\title{
Lightweight Authentication Protocol in Edge-based Smart Grid Environment
}

\author{
Chien-Ming Chen ${ }^{1}$, Lili Chen ${ }^{1}$, Yanyu Huang ${ }^{2}$, Sachin Kumar ${ }^{3}$ and Jimmy Ming-Tai Wu ${ }^{1 *}$
}

${ }^{*}$ Correspondence: wmt@wmt35.idv.tw

${ }^{1}$ Shandong University of Science and Technology, Shandong, China Full list of author information is available at the end of the article

\begin{abstract}
A smart grid (SG) is an advanced power grid system deployed in a cloud center and smart meters (at the consumer end) that provides higher reliability, better data protection, improved power efficiency, automatic monitoring, and effective management of power consumption. However, an SG also poses certain challenges that need to be addressed. For example, data provided by a smart meter are time sensitive and cannot handle high latency in an SG. Moreover, a smart meter depends on the memory, energy, and other factors. In addition, the security between a cloud center and smart meter is an important issue that needs to be resolved. Edge computing, which is an extension of cloud computing deployed in an edge network between a cloud center and the end devices, is an efficient solution to the above-mentioned issues. Therefore, in this study, we propose a secure mutual authentication protocol based on edge computing for use in an SG.
\end{abstract}

Keywords: smart grid; edge computing; mutual authentication; network security

\section{Introduction}

A traditional power grid provides four main operations: power generation, electricity transmission, electricity distribution, and electricity management. Currently, with the rapid development of IoT, the demand for electricity has significantly increased; however, the infrastructure used by traditional grids cannot sustain such high electricity demands. To solve this problem, a smart grid (SG) was developed. It is well-known that an SG is an advanced electricity grid that uses a two-way flow of electricity and information, which differs from a traditional electricity infrastructure, providing more efficiency, protected data submissions, and a secure channel between a smart meter (SM) and service provider (SP). The remarkable advantages of an SG include monitoring the electricity consumption of the endusers with different approaches in real time[1]. In general, the infrastructure of an SG comprises three components: in-house deployed SMs, SPs in substations, and a control center (CC) in the cloud[2, 3, 4, 5, 6]. The infrastructure of an SG is shown in Fig. 1. SMs are used for monitoring the energy consumption of consumers and sending the processed data to the SP, which likely contains private information of the consumers. SPs offer services and electricity to consumers. The CC processes, handles, and manages the resources of the sensors, SMs, and actuators, and stores the data in a data center for cloud computing. However, with an increasing number of IoT (end-user) devices, an SG based on cloud computing is unable to meet such requirements.

Conversely, in 2012, Cisco specified certain disadvantages of cloud computing, including a high latency, low mobility support, and low location awareness. As a 


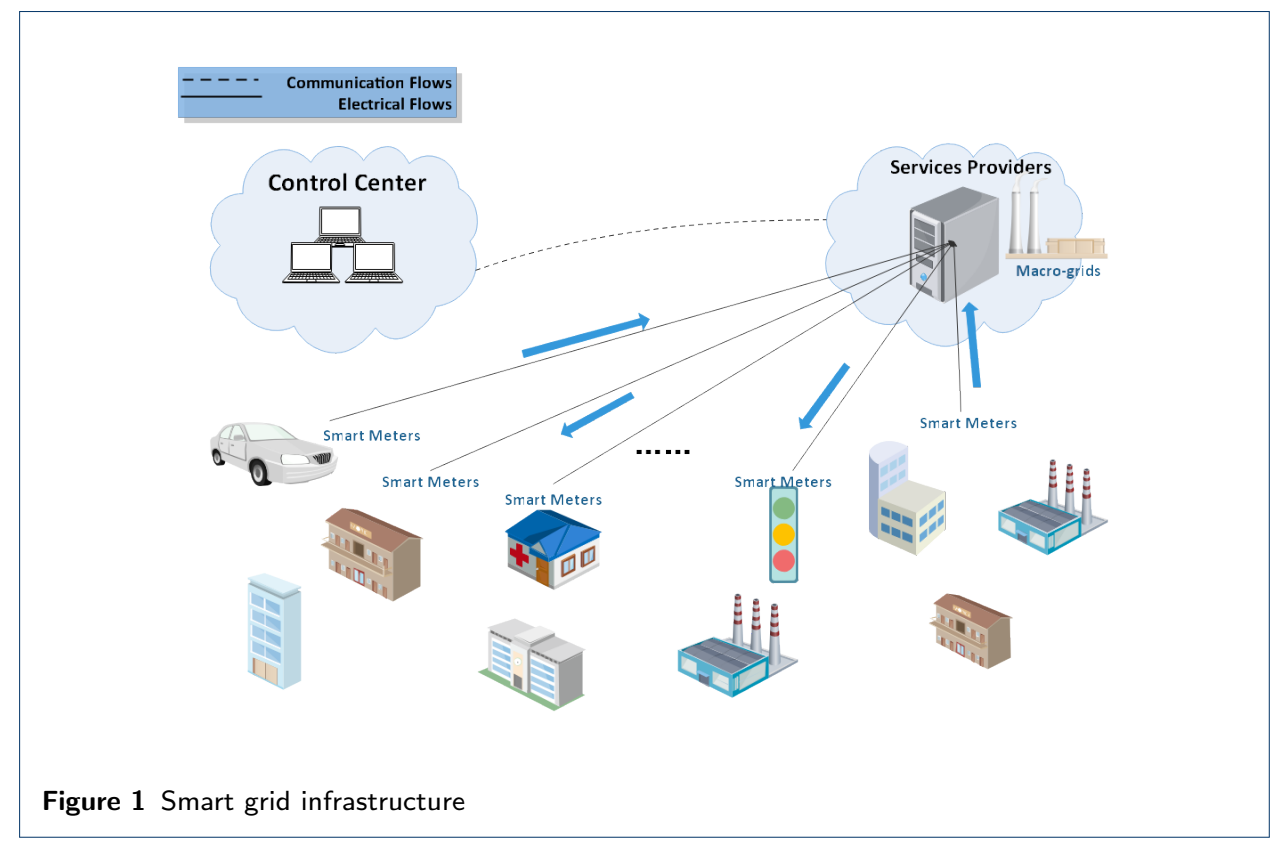

result, the company proposed the concept of fog computing, which is a type of edge computing as previously mentioned, as an extension of cloud computing[7]. An edge layer is employed between the end devices and the cloud center; in addition, each end device is directly connected to the edge nodes, and the edge nodes are interconnected, each linked to the cloud [8]. The edge nodes in edge computing consist of certain devices with limited computation power, such as switches, routers, mobile devices, and idle servers. The main role of an edge node is to collect and process the data from the end devices, issue control commands to the actuators, locally filter the data, and send the remaining data to the cloud center[9, 10]. Edge computing has the following characteristics. First, with the rapid development of mobile devices, it is important for the edge nodes to directly communicate with these devices, such as mobile phones, mobile sensors, and moving cars. Second, the batch process used in cloud computing cannot facilitate real-time interactions; however, with large numbers of edge nodes deployed in distributed locations, edge computing can provide real-time interactions. Third, the edge nodes used in edge computing are distributed in different places. Although the computational abilities of the edge nodes are limited, use of a large number of nodes can solve this problem, for example, by using an SG or a smart vehicle network. Finally, in edge computing, the edge nodes are deployed in different places closer to the end-users. Cloud computing applies a centralized architecture, in sharp contrast to the distributed edge architecture.

As previously mentioned, edge computing is an extension of cloud computing, which provides numerous advantages over the cloud computing infrastructure. In recent years, many researchers have attempted to extend the edge computing infrastructure for applications based on cloud computing $[1,11,12,13,14]$. In this study, we extended the infrastructure of edge computing to an SG, which is different from the case of a traditional SG. The advantage of an edge-computing based SG over a 
traditional SG includes minimum latency, providing services to resource-constrained devices, reduced stress on the cloud center, and preprocessing of unimportant data.

It is necessary to extend edge computing to an SG; however, the deployment of an SM is unsafe, and the meter needs to be protected by a physical lock to avoid a possible attack by an adversary. An adversary can obtain the data stored in an SM and pose as an SG to communicate with the SPs or consumers. Therefore, secure communication between the SG and SP is extremely important. A key agreement and mutual authentication protocol are efficient solutions to solving this problem. Several studies have also proposed protocols related to SGs[15, 16, 17, 18, 19]. However, a mutual authentication protocol for an SG based on the use of edge computing has yet to be proposed. An SG requires real-time data transmission. Addition of edge nodes to an SG can guarantee a low latency and real-time data response. We therefore propose a protocol based on edge computing for use in such a grid.

Contributions This study designed a secure and lightweight key exchange and mutual authentication protocol for an edge-based SG environment that includes the registration phase of the SMs and that of the edge nodes along with a mutual authentication. The edge node in this protocol is an authenticated node, which can authenticate the SMs, and generate a session key with, and provide services to, the SMs. In consideration of the constrained resources of the SMs and edge nodes, we used a one-way hash function, XOR computations, and an elliptic curve cryptosystem (ECC) instead of another cryptography method. In addition, in this study, we used an automatic cryptographic protocol tool, ProVerif, and Burrows-AbadiNeedham (BAN) logic to guarantee the security of our protocol, which also undergoes a verification stage. Our proposed protocol can provide perfect forward secrecy and withstand a replay attack, SM impersonation attack, edge node impersonation attack, off-line password guessing attack, man-in-the-middle attack, ephemeral secret leakage attack, and SM anonymity and traceability attack. In addition, we present a performance evaluation of our protocol to calculate the computational costs .

Organization The remainder of this paper is organized as follows. Section 2 briefly presents the recent studies related to the security of an SG. In section 3, we present an adversary model. The notations and details of the proposed protocol are presented in section 4 . To establish the security of the proposed protocol, a security analysis is presented in section 5, concluding with a formal security analysis using ProVerif and BAN logic, along with an informal security analysis; a comparison of the computation time of the proposed protocol with that of other SG-based protocols without an edge computing infrastructure is also provided. Finally, some concluding remarks are presented in section 6 .

\section{Related literature}

With the emergence of an SG in 2001, numerous researchers have worked on ensuring security in an SG. Hassan et al. [20] encountered several problems with the use of an SG. For instance, owing to uncertainties in the system planning and maintenance, it is difficult to predict real-time system controls. In addition, communication between system operators in a CC is another problem that needs to be considered. 
Moreover, lack of predictive control signals for operating the devices and lack of energy storage devices also affect the deployment of SMs.

In 2010, Ericsson [21] pointed out that the important aspects regarding the infrastructure of an SG are cyber security and power system communication (PSC). In addition, information security has become increasingly important because the deployment occurs in an exoteric and integrated energy management system instead of through isolated automation as previously applied. Moreover, with the development of the Internet, attackers can steal data from an SM and a cloud center.

To ensure the information security of an SG, researchers have proposed several secure mechanisms. Kim et al. [22] proposed a secure mechanism based on an SG according to the security requirements of remote meters using power-line communication (PLC), including authentication and key sharing between devices, as well as revocation management of the remaining devices. However, the SM server used in this mechanism demands authentication of all nodes, which may cause heavy stress on the SM server. When numerous devices are added in an SG environment, the mechanism will become overburdened.

For the purpose of efficient resource management and information security, some researchers have begun adding edge computing to an SG; for instance, Zahoor et al. [1] introduced a new SG model based on edge computing for resource management. The proposed model is based on an edge-cloud hierarchical infrastructure to separate the role of the cloud, providing different types of services to consumers. Compared with a traditional SG model, the proposed approach can improve the response time for for effective resource utilization and reduce the latency. In 2016, Nazmudeen et al. [23] proposed a distributed data aggregation method based on an edge computing architecture, limiting the amount of data sent to the centralized storage space, thereby improving the capacity of the PLC without affecting its functionality.

Although edge computing solves the problems inherent to cloud computing, information security, i.e., the security of a transmitted message through an unsecure channel, is of vital importance in an SG. A mutual authentication protocol can guarantee the security of inter-communication. In recent years, some mutual authentication protocols have been proposed to guarantee the security between parties $[24,25,26,27]$.

Zhang et al.[18] designed an authentication protocol based on elliptic curve cryptography for an SG, which can provide privacy protection. The authors claimed that the protocol has the advantages of identity protection, mutual authentication, and key agreement. However, after our analysis of the protocol, we found that it cannot resist an impersonation attack on an SM or SP or a replay attack. In addition, Tsai et al.[17] proposed a new anonymous key distribution scheme in an SG environment using identity-based signature schemes and identity-based encryption schemes. The advantages of the scheme include a trusted authority separate from the authentication phases and direct access of an SM to the SP without a trusted authority, which can lower the computation time. However, the proposed protocol is still vulnerable, cannot withstand a privileged insider attack, and provides imperfect forward secrecy. 


\section{Method}

In this section, we first introduce the infrastructure of smart grid based on edge computing. Then, we describe the adversary model used in this paper.

\subsection{Edge computing infrastructure for smart grid}

In this study, we proposed a protocol based on edge computing for an SG. Our SG infrastructure based on edge computing is shown in Fig. 2. An edge layer is used to join the infrastructure, acting as an SP in an SG. The cloud is separated to handle data from the edge layer and transmit them to the CC. This infrastructure using different SPs from the macro-grids, which can reduce the burden of the cloud, integrates the main capacities of the cloud to communicate with the CC and management control.

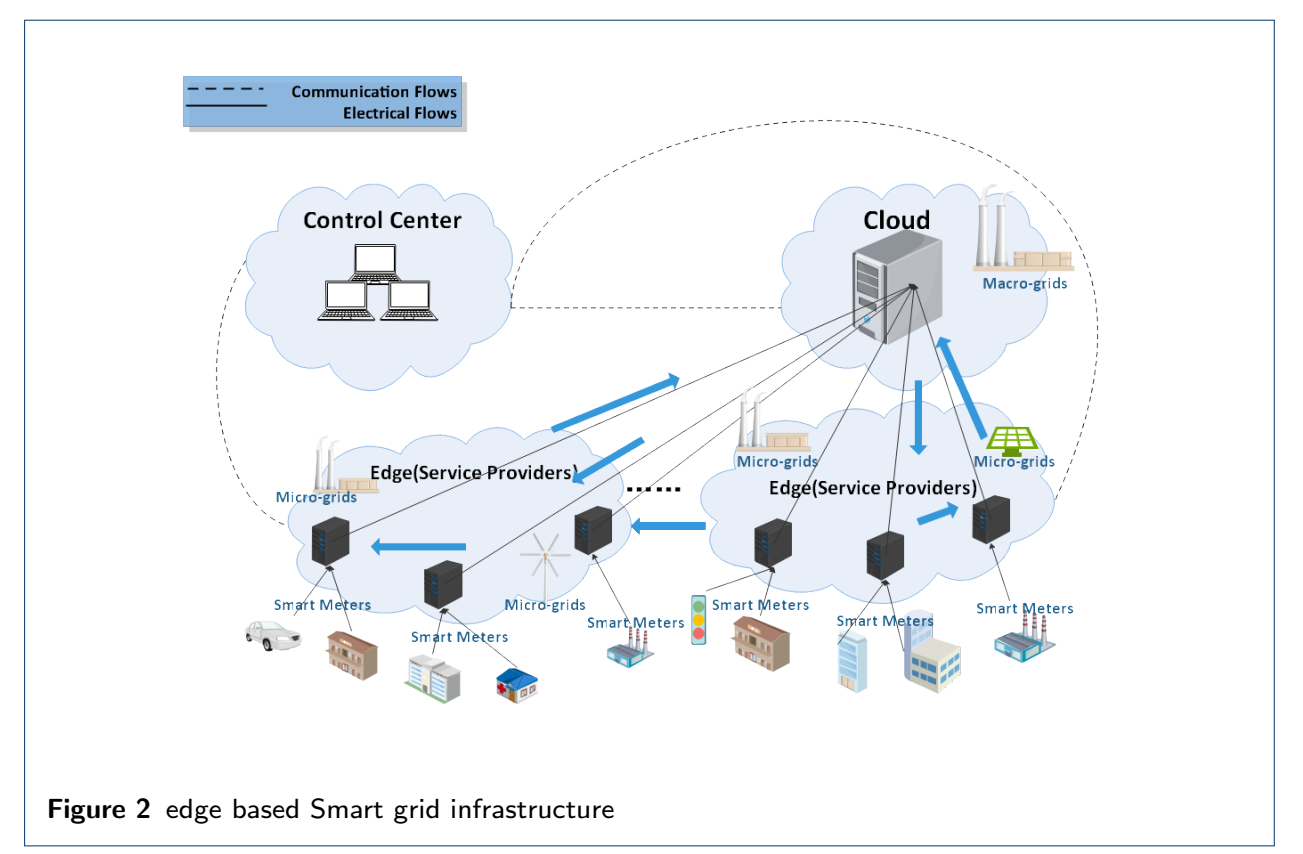

In our protocol, edge nodes act as SPs that can quickly process the data and authenticate an SM. Because of the limited computations of the edge nodes and SM, the proposed protocol only uses an ECC and one-way hash function for encrypting the parameters. Our protocol comprises the following phases.

\subsection{Adversary model}

Before introducing our proposed protocol, it is important to describe the adversary model applied. Assuming the presence of an adversary over the Internet, the adversary model, $A d v$, is defined as follows:

- Adv can control limited/completed messages transmitted over an insecure channel, such as intercepting, modifying, and deleting the transmitted message.

- $A d v$ can extract the security parameters stored in a smart card using a power analysis technique.

- $A d v$ can try to obtain sensitive information (e.g., passwords) using off-line password guessing attacks. 
Table 1 Notations used in the proposed protocol

\begin{tabular}{ll}
\hline Notations & Descriptions \\
\hline$S M$ & Smart Meter \\
$E S_{j}$ & $j^{\text {th } \text { edge node }}$ \\
$T A$ & Trusted Authority \\
$E_{p}(a, b), p$ & Elliptic curve cryptosystem, $E_{p}: y^{3}=x^{3}+$ \\
& $a x+b(\bmod p)$ is an elliptic curve over prime \\
& field $F_{p}, p$ is a prime, where $x, y, a, b \in F_{p}$ and \\
& $\left(4 a^{3}+27 b^{2}\right) \bmod p \neq 0$ \\
$k G$ & $k G=G+G+\ldots+G\left(k t i m e s, k \in F_{p}\right)$, Scalar \\
& multiplication in Elliptic Curve Cryptosystem. \\
$r_{i}$ & The register parameters generated by Trusted \\
$A d v$ & Authority \\
$s$ & Adversary \\
$S K_{i j}, S K_{j i}$ & Secret Key of $T A$ \\
$n_{i}, n_{j}$ & Session Key by SMs and edge nodes \\
$H(\cdot)$ & random number chosen by SMs and edge nodes \\
$\|$ & Cryptographic one-way hash function \\
$\oplus$ & Concatenation \\
\hline
\end{tabular}

\section{Proposed protocol}

Herein, we describe the proposed protocol, which consists of three phases, an edge node registration phase, an SM registration phase, and a login and authentication phase. Table 1 summarizes the notations used in our proposed protocol.

\subsection{Edge node registration phase}

If an edge node $E S_{j}$ wants to join the system, the edge node registration phase is applied. This phase is shown in fig4 and is described as follows:

(i) $E S_{j}$ first selects an identity $S I D_{j}$ and transmits $\left\{S I D_{j}\right\}$ to $T A$ through a secure channel.

(ii) After receiving the above messages, $T A$ checks the validity of $E S_{j}$. Then, $T A$ computes $R S I D_{j}=H\left(S I D_{j} \| s\right)$, stores $\left\{S I D_{j}, R S I D_{j}\right\}$ in the database of $T A$, and transmits $\left\{R S I D_{j}\right\}$ back to $E S_{j}$ through a secure channel.

(iii) $E S_{j}$ stores $\left\{R S I D_{j}\right\}$ in its database.

\subsection{Smart meter registration phase}

The SM registration phase (fig3) is executed if an $S M$ registers with $T A$. We assume that an $S M$, whose identity is $I D_{i}$, wants to join this system, and there are $n$ edge nodes, denoted as $E S_{1}, \ldots, E S_{n}$, that have been previously registered. The following steps are conducted.

(i) $S M$ selects identity $I D_{i}$ and a random number $r_{i}$, and then transmits $\left\{I D_{i}, r_{i}\right\}$ to the trusted authority $T A$ through a secure channel.

(ii) After receiving the message from $S M, T A$ first finds all values of $R S I D_{j}$ stored in the database where $1 \leq j \leq n$. Next, $T A$ computes $A_{j}=$ $H\left(I D_{i} \| R S I D_{j}\right), B A u_{j}=A_{j} \oplus H\left(I D_{i} \| r_{i}\right), C_{j}=H\left(S I D_{j} \| R S I D_{j}\right)$ and $D A u_{j}$ $=C_{j} \oplus H\left(I D_{i} \| r_{i}\right)$, and then transmits $\left\{S I D_{j}, B A u_{j}, D A u_{j}, H(\cdot)\right\}$ back to $S M$ through a secure channel.

(iii) The $S M$ computes $S I D_{j}^{*}=S I D_{j} \oplus H\left(I D_{i} \| r_{i}\right), B A u_{j}^{*}=B A u_{j} \oplus H\left(I D_{i} \| r_{i}\right)$, $D A u_{j}^{*}=S I D_{j} \oplus B A u_{j} \oplus D A u_{j}$ and stores $\left\{S I D_{j}^{*}, B A u_{j}^{*}, D A u_{j}^{*}, H(\cdot)\right\}$ into memory. 
SMS

Select $I D_{i}, r_{i}$

$B A u_{j}^{*}=B A u_{j} \oplus H\left(I D_{i} \| r_{i}\right)$,

$D A u_{j}^{*}=S I D_{j} \oplus B A u_{j} \oplus D A u_{j}$

Stores $\left\{S I D_{j}^{*}, B A u_{j}^{*}, D A u_{j}^{*}\right\}$
$T A$

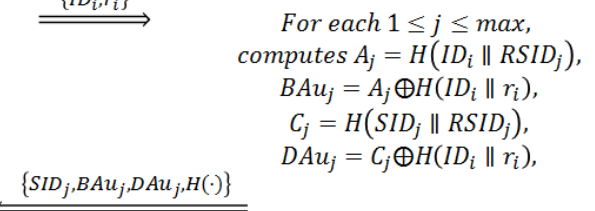

For each $1 \leq j \leq \max$, computes $A_{j}=H\left(I D_{i} \| R S I D_{j}\right)$, $B A u_{j}=A_{j} \oplus H\left(I D_{i} \| r_{i}\right)$, $C_{j}=H\left(S I D_{j} \| R S I D_{j}\right)$, $D A u_{j}=C_{j} \oplus H\left(I D_{i} \| r_{i}\right)$,

$\left\{S I D_{j}, B A u_{j}, D A u_{j}, H(\cdot)\right\}$

Secure channel

Figure 3 Smart meter registration phase

$$
E S_{j}(S M)
$$

Select $S I D_{j}$

Stores $\left\{\operatorname{RSID}_{j}\right\}$
TA

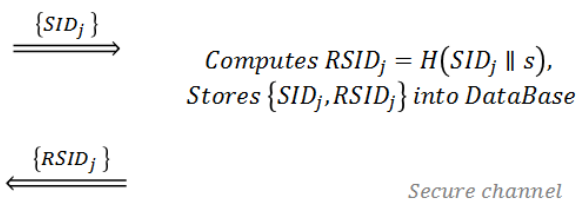

Figure 4 Edge node registration phase

\subsection{Login and authentication phase}

When a legal $S M$ wants to $\log$ in and communicate with $E S_{j}$, the $S M$ needs to authenticate $E S_{j}$ and establish a session key with $E S_{j}$ using the following steps.

(i) The $S M$ first enters its identity $I D_{i}$ and $r_{i}$, and then computes $S I D_{j}=$ $S I D_{j}^{*} \oplus H\left(I D_{i} \| r_{i}\right), B A u_{j}=B A u_{j}^{*} \oplus H\left(I D_{i} \| r_{i}\right), D A u_{j}=S I D_{j} \oplus B A u_{j} \oplus$ $D A u_{j}^{*}$, and $C_{j}=D A u_{j} \oplus H\left(I D_{i} \| r_{i}\right)$. Next, $S M$ generates the current timestamp $T_{i}$ and calculates $E_{i}=I D_{i} \oplus H\left(C_{j} \| T_{i}\right)$. In addition, $S M$ then generates a random number $n_{i}$, computing $N_{i}=n_{i} P, A_{j}=B A u_{j} \oplus H\left(I D_{i} \| r_{i}\right)$, $F_{i}=H\left(I D_{i} \| A_{j}\right), G_{i}=F_{i} \oplus N_{i}$, and authentication parameter $M_{1}=$ $H\left(I D_{i}\left\|F_{i}\right\| N_{i} \| T_{i}\right)$, sending $\left\{E_{i}, G_{i}, M_{1}, T_{i}\right\}$ to $E S_{j}$ through an unsecure channel.

(ii) After receiving the message from $S M, E S_{j}$ calculates $C_{j}^{\prime}=H\left(S I D_{j} \| R S I D_{j}\right)$, $I D_{i}^{\prime}=E_{i} \oplus H\left(C_{j}^{\prime} \| T_{i}\right), A_{j}^{\prime}=H\left(I D_{i}^{\prime} \| R S I D_{j}\right), F_{i}^{*}=H\left(I D_{i}^{\prime} \| A_{j}^{\prime}\right), N_{i}^{\prime}=$ $F_{i}^{\prime} \oplus G_{i}$, and $M_{1}^{\prime}=H\left(I D_{i}^{\prime}\left\|F_{i}^{\prime}\right\| N_{i}^{\prime} \| T_{i}\right)$, and then checks the validity of $M_{1}$ to verify if $S M$ is legal. If so, $E S_{j}$ generates a random number $n_{j}$ and current timestamp $T_{j}$, and then computes $N_{j}=n_{j} P, M_{2}=H\left(A_{j}^{\prime}\left\|C_{j}^{\prime}\right\| N_{j} \| T_{j}\right), I_{j}=$ $H\left(C_{j}^{\prime}\right) \oplus N_{i}^{\prime}, K_{j}=I_{j} \oplus N_{j}$, and the session key $S K_{j i}=H\left(I D_{i}^{\prime}\left\|A_{j}^{\prime}\right\| n_{j} N_{i}^{\prime}\right)$, and transmits the message $\left\{M_{2}, K_{j}, T_{j}\right\}$ to $S M$.

(iii) When $S M$ receives the messages from $E S_{j}, S M$ first computes $I_{j}^{\prime}=H\left(C_{j}\right) \oplus$ $N_{i}, N_{j}^{\prime}=I_{j}^{\prime} \oplus K_{j}$, and $M_{2}^{\prime}=H\left(A_{j}\left\|C_{j}\right\| N_{j}^{\prime} \| T_{j}\right)$, and then checks the valid- 


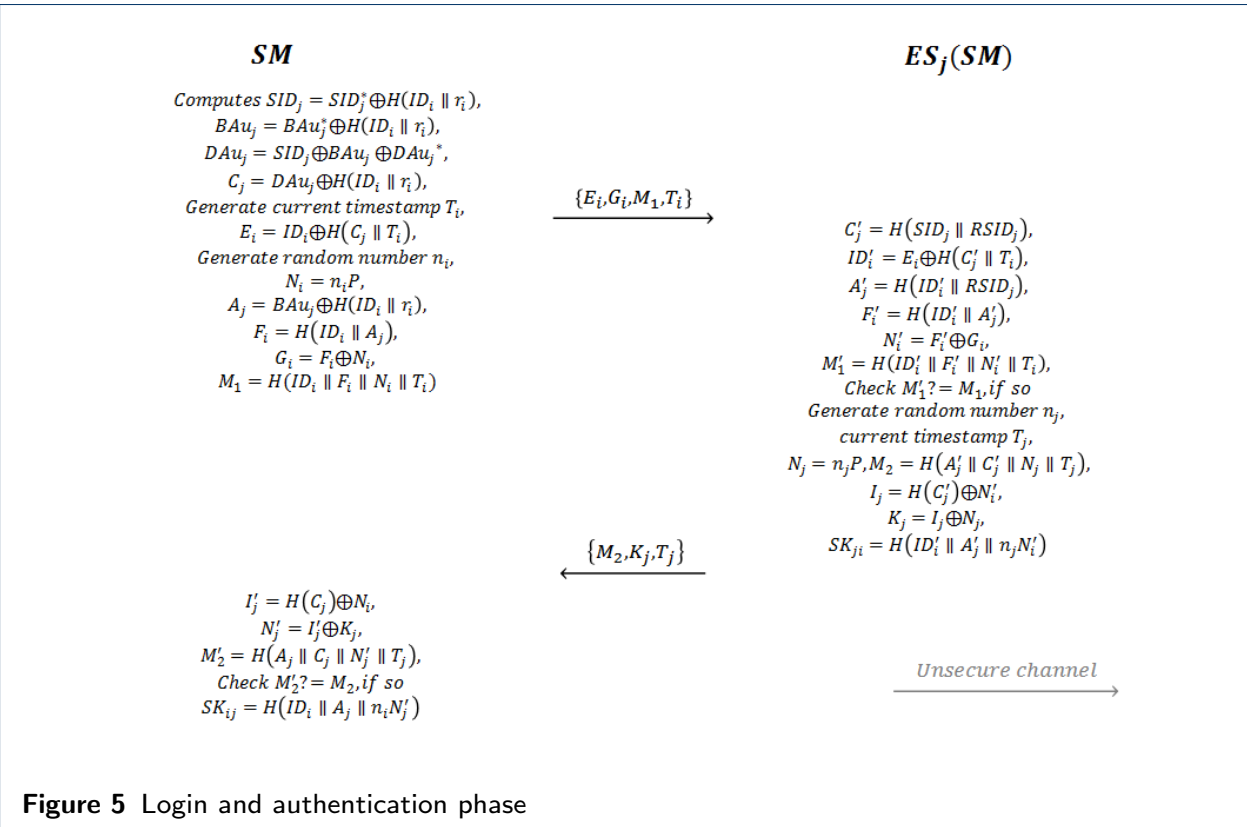

Figure 5 Login and authentication phase

ity of $E S_{j}$ by checking whether $M_{2}^{\prime}=M_{2}$. If so, $S M$ computes the session key $S K_{i j}=H\left(I D_{i}\left\|A_{j}\right\| n_{i} N_{j}^{\prime}\right)$, which means that $S M$ and $E S_{j}$ can securely communicate with each other.

The SM login and authentication phase is illustrated in Fig. 5.

\section{Security analysis of the proposed protocol}

In this section, we evaluate the security of the proposed protocol with BAN logic and an an automatic cryptographic tool called ProVerif.

\subsection{Formal security analysis using BAN logic}

In this subsection, we demonstrate the security of our solution during the authentication phase through the BAN logic. In this study, the user $(S M)$ and the edge node $E S_{j}$ authenticate each other and calculate a session key. Below are some of the symbols and rules defined when using the BAN logic.

\subsubsection{Notations used in BAN logic}

- $P \mid \equiv X$ : The principal $\mathrm{P}$ believes $\mathrm{X}$, or is entitled to do so. In particular, $\mathrm{P}$ may act as though $\mathrm{X}$ is true. This construct is central to the logic.

- $P \triangleleft X$ : $\mathrm{P}$ sees $\mathrm{X}$. Someone sends a message containing $\mathrm{X}$ to $\mathrm{P}$, who can read and repeat $\mathrm{X}$ (possibly after a decryption).

- $P \mid \sim X$ : $\mathrm{P}$ once stated $\mathrm{X}$. At some point of time, $\mathrm{P}$ sent a message including statement X. It is unknown whether the message was sent long ago or during the current run of the protocol, but it is known that $\mathrm{P}$ believed $\mathrm{X}$ at that time.

- $P \mid \Longrightarrow X$ : $\mathrm{P}$ has jurisdiction over $\mathrm{X}$. The principal $\mathrm{P}$ is an authority on $\mathrm{X}$ and should be trusted in this matter. For example, a server is often trusted to properly generate encryption keys. This may be expressed based on the 
assumption that the principals believe that the server has jurisdiction over statements regarding the quality of these keys.

- $\sharp(X)$ : The formula $\mathrm{X}$ is fresh; that is, $\mathrm{X}$ has not been sent in a message at any time before the current run of the protocol. This is typically true for a nonce, that is, an expression invented for the purpose of being fresh. A nonce commonly includes a timestamp or number that is used only once.

- $P \stackrel{K}{\longleftrightarrow} Q$ : $\mathrm{P}$ and $\mathrm{Q}$ may use a shared key $\mathrm{K}$ to communicate. Key $\mathrm{K}$ is safe in that it will never be discovered by any principal except $\mathrm{P}$ or $\mathrm{Q}$, or by a principal trusted by either $\mathrm{P}$ or $\mathrm{Q}$.

- $P \stackrel{X}{\rightleftharpoons} Q$ : Formula $\mathrm{X}$ is a secret known only to $\mathrm{P}$ and $\mathrm{Q}$ and possibly to principals trusted by them. Only $\mathrm{P}$ and $\mathrm{Q}$ may use $\mathrm{X}$ to prove their identities to one another. An example of a secret is a password.

- $\{X\}_{K}$ : Formula $\mathrm{X}$ is encrypted under key K.

- $\langle X\rangle_{Y}$ : Formula $\mathrm{X}$ is combined with formula $\mathrm{Y}$.

\subsubsection{BAN logic rules}

(i) The message-meaning rule for shared keys is $\frac{P \mid \equiv P \stackrel{K}{\longleftrightarrow} Q, P \triangleleft\{X\}_{K}}{P|\equiv Q| \sim X}$. This indicates that, if $\mathrm{P}$ believes that $\mathrm{K}$ is a key shared with $\mathrm{Q}$ and if $\mathrm{P}$ sees $\mathrm{X}$ encrypted under $\mathrm{K}$, then $\mathrm{P}$ believes that $\mathrm{Q}$ once stated $\mathrm{X}$.

(ii) The message-meaning rule for shared secrets: $\frac{P \mid \equiv P \stackrel{Y}{\rightleftharpoons} Q, P \triangleleft\langle X\rangle_{Y}}{P|\equiv Q| \sim X}$. This means that, if $\mathrm{P}$ believes that $\mathrm{Y}$ is a secret known only to $\mathrm{P}$ and $\mathrm{Q}$ and $\mathrm{P}$ sees $\mathrm{X}$ under $\mathrm{Y}$, then $\mathrm{P}$ believes that $\mathrm{Q}$ once stated $\mathrm{X}$.

(iii) The nonce-verification rule is $\frac{P|\equiv \sharp(X), P| \equiv Q \mid \sim X}{P|\equiv Q| \equiv X}$. This means that, if $\mathrm{P}$ believes that $\mathrm{X}$ is fresh and $\mathrm{Q}$ once stated $\mathrm{X}$, then $\mathrm{P}$ believes that $\mathrm{Q}$ believes $\mathrm{X}$.

(iv) The jurisdiction rule is $\frac{P|\equiv Q| \Longrightarrow X, P|\equiv Q| \equiv X}{P \mid \equiv X}$. This means that, if $\mathrm{P}$ believes that $\mathrm{Q}$ has jurisdiction over $\mathrm{X}$ and believes that $\mathrm{Q}$ believes $\mathrm{X}$, then $\mathrm{P}$ believes $\mathrm{X}$.

(v) The session key rule is $\frac{P|\equiv \sharp(X), P| \equiv Q \mid \equiv X}{P \mid \equiv P \stackrel{K}{\longrightarrow} Q}$. This means that, if $\mathrm{P}$ trusts that statement formula $\mathrm{X}$ is fresh and $\mathrm{P}$ trusts that $\mathrm{Q}$ trusts $\mathrm{X}$, which is an essential component of the session key, then $\mathrm{P}$ trusts that he or she shares the session key K with Q.

(vi) The freshness rule is $\frac{P \mid \equiv \sharp(X)}{P \mid \equiv \sharp(X, Y)}$. This means that, if $\mathrm{P}$ believes that $\mathrm{X}$ is fresh, then he or she believes the freshness of $(\mathrm{X}, \mathrm{Y})$.

(vii) The belief rule is $\frac{P|\equiv X, P| \equiv Y}{P \mid \equiv(X, Y)}$. This means that, if $\mathrm{P}$ believes $\mathrm{X}$ and $\mathrm{Y}$, then $\mathrm{P}$ believes $(\mathrm{X}, \mathrm{Y})$.

5.1.3 Goals

- G1: $S M \mid \equiv S M \stackrel{S K}{\longleftrightarrow} E S_{j}$.

- G2: $E S_{j} \mid \equiv S M \stackrel{S K}{\longleftrightarrow} E S_{j}$.

- G3: $S M\left|\equiv E S_{j}\right| \equiv S M \stackrel{S K}{\longleftrightarrow} E S_{j}$.

- G4: $E S_{j}|\equiv S M| \equiv S M \stackrel{S K}{\longleftrightarrow} E S_{j}$.

\subsubsection{Idealize the communication messages}

- Meg1: $S M \rightarrow E S_{j}:\left\{E_{i}, G_{i}, M_{1}, T_{i}\right\}$.

- Meg2: $S_{j} \rightarrow S M:\left\{M_{2}, K_{j}, T_{j}\right\}$. 


\subsubsection{Initial state assumptions}

- A1: $S M \mid \equiv \sharp\left(n_{i}\right)$.

- A2: $E S_{j} \mid \equiv \sharp\left(n_{j}\right)$.

- A3: $S M \mid \equiv S M \stackrel{C_{j}}{\rightleftharpoons} E S_{j}$.

- $\mathbf{A} 4: S M\left|\equiv E S_{j}\right| \Longrightarrow N_{j}$.

- A5: $E S_{j} \mid \equiv S M \stackrel{H\left(C_{j} \| T_{i}\right)}{\rightleftharpoons} E S_{j}$.

- A6: $E S_{j} \mid \equiv \sharp\left(I D_{i}\right)$.

- A7: $E S_{j}|\equiv S M| \Longrightarrow I D_{i}$.

- A8: $S M \mid \equiv \sharp\left(N_{j}\right)$.

- A9: $E S_{j} \mid \equiv \sharp\left(N_{i}\right)$.

- A10: $E S_{j}|\equiv S M| \Longrightarrow N_{i}$.

\subsubsection{Main proofs using BAN rules and assumptions}

Based on the BAN logic rules, we demonstrate that the proposed key exchange protocol can use the initial state assumptions to achieve the defined goals. Below are the steps used to prove the BAN logic.

For G1, according to the message Meg2 and using the seeing rule, we obtain S1: $S M \triangleleft\left\{M_{2}:\left\langle A_{j}, N_{j}, T_{j}\right\rangle_{C_{j}} ; K_{j}, T_{j}\right\}$. Using A3, S1, and the message-meaning rule, we obtain S2: $S M\left|\equiv E S_{j}\right| \sim\left(A_{j}, N_{j}, T_{j}\right)$. Using A3 and S2, and applying the freshness and nonce-verification rules, S3: $S M\left|\equiv E S_{j}\right| \equiv\left(A_{j}, N_{j}, T_{j}\right)$ is obtained. Applying the belief rule for each component, we obtain $\mathbf{S} 4: S M\left|\equiv E S_{j}\right| \equiv N_{i}$. Using A4, S4, and the jurisdiction rule, S5: $S M \mid \equiv N_{j}$ is obtained. Because $S K=$ $H\left(I D_{i}\left\|A_{j}\right\| n_{i} N_{j}\right)$, we obtain S6: $S M \mid \equiv S M \stackrel{S K}{\longleftrightarrow} E S_{j}$.

For G2, according to the message Meg1 and using the seeing rule, we obtain S7: $E S_{j} \triangleleft\left\{E_{i}:\left\langle I D_{i}\right\rangle_{H\left(C_{j} \| T_{i}\right)} ; G_{i}, M_{1}:\left\langle F_{i}, N_{i}, T_{i}\right\rangle_{I D_{i}} ; T_{i}\right\}$. Using the seeing rule for each component, we obtain S8, i.e., $E S_{j} \triangleleft\left\{\left\langle I D_{i}\right\rangle_{H\left(C_{j} \| T_{i}\right)}\right\}$, and S9, i.e., $E S_{j} \triangleleft$ $\left\{\left\langle F_{i}, N_{i}, T_{i}\right\rangle_{I D_{i}}\right\}$. Using A5, S8, and the message-meaning rule, we obtain S10: $E S_{j}|\equiv S M| \sim I D_{i}$. Using $\mathrm{A} 6$ and $\mathrm{S} 10$ and applying the nonce-verification rule, S11: $E S_{j}|\equiv S M| \equiv I D_{i}$ is obtained. Using A7, S11, and the jurisdiction rule, we obtain S12: $E S_{j} \mid \equiv I D_{i}$. Using A6, S11, and the session key rule, we obtain S13: $E S_{j} \mid \equiv S M \stackrel{I D_{i}}{\rightleftharpoons} E S_{j}$. Using S9, S13, and the message-meaning rule, we obtain S14: $E S_{j}|\equiv S M| \sim\left(F_{i}, N_{i}, T_{i}\right)$. According to A9 and S15 and using the freshness and nonce-verification rules, S15: $E S_{j}|\equiv S M| \equiv\left(F_{i}, N_{i}, T_{i}\right)$ is obtained. Based on the belief rule, we obtain S16: $E S_{j}|\equiv S M| \equiv N_{i}$. Using A10, S16, and the jurisdiction rule, we obtain S17: $S M \mid \equiv N_{i}$. Because $A_{j}=H\left(I D_{i} \| R S I D_{j}\right)$ and $S K=H\left(I D_{i}\left\|A_{j}\right\| n_{j} N_{i}\right)$, we obtain S18: $E S_{j} \mid \equiv S M \stackrel{S K}{\longleftrightarrow} E S_{j}$.

Applying the belief rule for each component, we obtain $\mathbf{S} 4: S M\left|\equiv E S_{j}\right| \equiv N_{i}$. Using A4, S4, and the jurisdiction rule, $\mathbf{S} 5: S M \mid \equiv N_{j}$ is obtained. Because $S K=$ $H\left(I D_{i}\left\|A_{j}\right\| n_{i} N_{j}\right)$, we obtain S6: $S M \mid \equiv S M \stackrel{S K}{\longleftrightarrow} E S_{j}$.

For G3, according to S6, A1, and the session key rule, we obtain S19: $S M \mid \equiv$ $E S_{j} \mid \equiv S M \stackrel{S K}{\longleftrightarrow} E S_{j}$.

For G4, according to S18, A2, and the session key rule, we obtain S20: $E S_{j} \mid \equiv$ $S M \mid \equiv S M \stackrel{S K}{\longleftrightarrow} F S_{j}$. 


\subsection{Formal security analysis using ProVerif tool}

As is well known, ProVerif is an automatic tool based on pi calculus. It focuses on the security of the cryptographic protocol[28], which is widely applied for formal security verification in numerous key exchange and mutual authentication protocols under a normal attack, including symmetric and asymmetric encryption, hash functions, digital signatures, and bit commitment. Therefore, we use ProVerif to establish the security of our proposed protocol. The resulting list after verification is shown in Fig. 12.

- First, we define a secure channel sch used for the registration of the SMs and edge nodes, applying a public channel $c h$ for login and authentication. The SMs and edge nodes generate session keys $S K_{i j}$ and $S K_{j i}$, respectively, and the functions and rules are then applied (Fig 7).

- We then verify the security of the proposed protocol using the following three queries. The former two consider whether an adversary has shared session keys, and the latter one verifies the correctness of the authentication process (Fig 8).

- The process of the main execution (Fig 6).

- The process of the edge nodes (Fig 9).

- The process of the SMs (Fig 10).

- The process of the trusted authority (Fig 11).

$$
\begin{aligned}
& \left({ }^{*}\right. \text { - Main - *) } \\
& \text { process } \\
& \quad \text { (IProcessUi [ I ProcessTA | IProcessES) }
\end{aligned}
$$

Figure 6 Main execution

\section{Discussion}

In this section, we further discuss our protocol can resist such various attacks. First, we assume that the adversary is represented as $A d v$.

Replay attack A replay attack resends the messages intercepted by $A d v$, which can obtain the messages of $\left\{E_{i}, G_{i}, M_{1}, T_{i}\right\}$ and $\left\{M_{2}, K_{j}, T_{j}\right\}$. We can see that they all have a timestamp in every transmitted message, which guarantees the freshness of the messages; timestamp $T_{i}$ and $T_{j}$ are both used in a later authentication parameter to check the validity of each other. Therefore, a faked timestamp cannot pass the verification stage. As a result, an adversary cannot replay the messages, and our protocol effectively resists a replay attack.

\section{SMs and edge node impersonation attack}

If adversary $A d v$ wants to create a login message $\left\{E_{a}, G_{a}, M_{1 a}, T_{a i}\right\}$ or $\left\{M_{2 a}, K_{a}, T_{a j}\right\}$ to pose as a legal SM $S M$ or legal $E S_{j}$. Taking $S M$ as an example, $E S_{j}$ is similar to $S M$. If $A d v$ wants to $\log$ into $E S_{j}$, he or she first needs the parameters of $C_{j}$ to calculate $I D_{i}$; however, without the knowledge of $S I D_{j}$ and $R S I D_{j}, A d v$ cannot obtain $C_{j}$ without $I D_{i}$, and $A d v$ cannot calculate $F_{i}$ and $N_{i}$. Therefore, it is impossible for $A d v$ to create a legal login message, and hence, our protocol can resist attacks on SMs and edge node impersonation attacks.

Man-in-middle attack As mentioned previously, $A d v$ cannot obtain the messages of both $\left\{E_{a}, G_{a}, M_{1 a}, T_{a i}\right\}$ and $\left\{M_{2 a}, K_{a}, T_{a j}\right\}$, and thus $A d v$ cannot forge 
$\left({ }^{*}\right.$ channel*)

free ch:channel. (* public channel *)

înee sch:channel [private]. (* secure channel, used for registering *)

(* shared keys *)

free SKij:bitstring [private].

free SKji:bitstring [private].

(* constants *)

free s:bitstring [private]. [(*the TA's secret key *)|

free RSIDj:bitstring[private].

free SIDj:bitstring[private].

const P:bitstring. (* the point on ecc *)

const G:bitstring. (* the generator *)

(* functions \& reductions \& equations *)

fun $\mathrm{H}$ (bitstring): bitstring. (* hash function*)

fun mult(bitstring,bitstring):bitstring. (* scalar multiplication operation *)

fun mod(bitstring,bitstring):bitstring. (* modulus operation *)

fun addone(bitstring):bitstring. ( ${ }^{*}$ add one *)

fun senc(bitstring,bitstring):bitstring. (* symmetric encryption *) reduc forall $m$ :bitstring, key:bitstring; $\operatorname{sdec}(\operatorname{sen} c(m, k e y), k e y)=m$.

fun con(bitstring,bitstring):bitstring. ( ${ }^{*}$ concatenation operation *)

reduc forall m:bitstring, $n$ :bitstring; getmess $(\operatorname{con}(m, n))=m$.

fun xor(bitstring,bitstring):bitstring. (* XOR operation*)

equation forall m:bitstring, n:bitstring; $x o r(x o r(m, n), n)=m$.

fun inverse(bitstring):bitstring. (*inverse operation*)

equation forall a:bitstring; inverse (inverse (a))= a.

fun gen(bitstring):bitstring. ( $\quad$ *Generator operation*)

fun rep(bitstring, bitstring):bitstring.

(* event *)

event UserStarted().

event UserAuthed().

Figure 7 Functions and rules

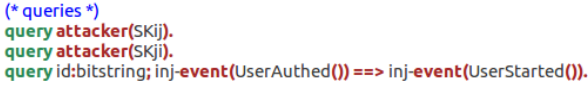

Figure 8 Three queries

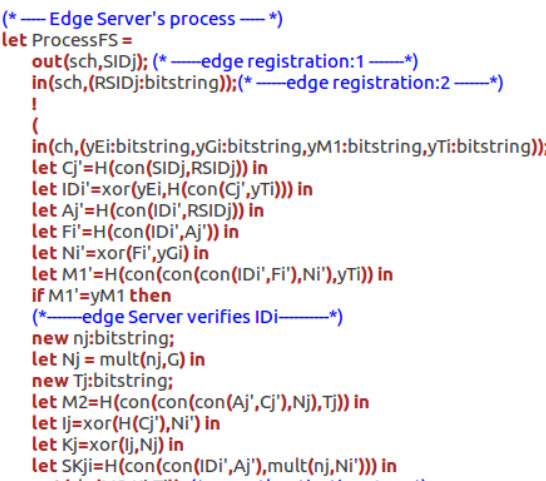

Figure 9 Process of edge nodes 


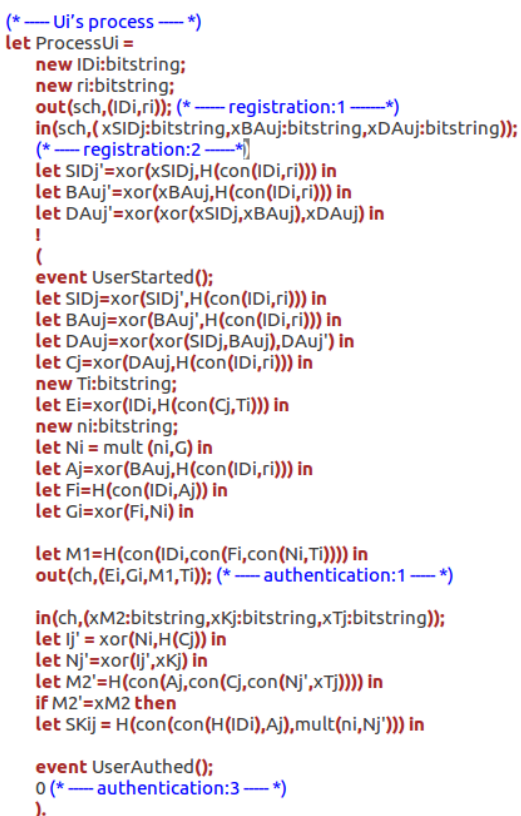

Figure 10 Process of smart meter

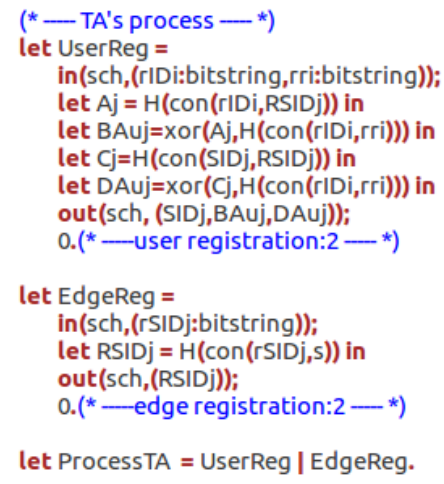

Figure 11 Process of a trusted authority

legal SMs or an edge node. Thus, our proposed protocol is resilient against a manin-the-middle attack.

Perfect forward secrecy In perfect forward secrecy, the long-term key $s$ indeterminable for $A d v$, and the messages over an insecure channel and the parameters from the memory of the SMs are revealed to $A d v$; however, even with these parameters, $A d v$ still cannot expose the session key between SMs and edge nodes. In our proposed protocol, if $A d v$ wants to calculate a session key $S K_{i j}=H\left(I D_{i}\left\|A_{j}\right\| n_{i} N_{j}^{\prime}\right)$, $A d v$ needs to know the parameters, $I D_{i}$ and $A_{j}$, and the random parameters, $n_{i} N_{j}$ or $n_{j} N_{i}$, whereas $I D_{i}, A_{j}, n_{i} N_{j}$, and $n_{j} N_{i}$ are independent of the long-term key and cannot be calculated based on messages from an insecure channel; therefore Adv cannot obtain the parameters used to compute the session key. Hence, our protocol can guarantee perfect forward secrecy. 


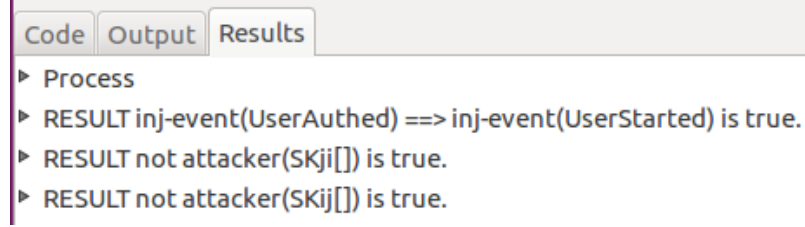

Figure 12 Formal security verification using ProVerif

Ephemeral secret leakage attack As mentioned above, if $A d v$ wants to obtain a session key, he or she needs to first obtain the ephemeral secret $n_{i}, n_{j}$. In an ephemeral secret leakage attack, $A d v$ can obtain the random parameters $n_{i}$ and $n_{j}$; however, if $A d v$ wants to obtain the session key, $A d v$ needs another two parameters $I D_{i}$ and $A_{j}$, which cannot be obtained from an insecure channel or the memory of the SMs. This proves that our proposed protocol is resilient against an ephemeral secret attack.

SM anonymity and untraceability attack In our protocol, the random numbers $n_{i}$ and $n_{j}$ and timestamp $T_{i}, T_{i}$ are used in the login and authentication phase. Different sessions have different messages; thus, $A d v$ cannot trace the message to focus on specific SMs, and the proposed protocol can have security against an SM anonymity attack. In addition, the identities of the SMs and edge nodes are masked by a random number and timestamp, which is also different, and hence our protocol can resist an untraceability attack.

\section{Experimental Result and Comparison}

In this section, some protocols related with an SG that do not employ edge computing, are listed and compared with our proposed protocol to prove the higher performance of the latter. To objectively analyze the protocols, we used an iPhone 7 for accurately determining the computational costs. In the evaluation based on experimental data, to evaluate the proposed protocol, we use $T_{h}, T_{d}, T_{p a}, T_{p m}$, $T_{a e}, T_{a d}, T_{e x p}$, and $T G_{e}$ to represent the time required for performing a one-way hash function, a symmetric decryption/encryption operation, an ECC point addition, a point multiplication, asymmetric public key encryption, asymmetric public key decryption, modular exponentiation, and a bilinear pairing operation. The time required for a bitwise XOR computation is negligible, and therefore, we do not consider the XOR computation time. Table 3 lists the computation time for these operations.

The computation times for the related SG protocols and the proposed protocol are presented in Table 3. A bar chart of the computation times is shown in Fig. 13. From the bar chart, it can be concluded that the proposed protocol involves the minimum computation time in all stages of entities. Although the computation time of Zhang et al.'s protocol was approximately $110.77 \mathrm{~ms}$, which is similar to that of the proposed protocol, Zhang et al.'s protocol cannot resist a privileged insider attack or provide perfect forward secrecy. Therefore, taking all requirements into account, our proposed protocol can provide easy computations and security against various attacks. 
Table 2 Computation time of protocols

\begin{tabular}{ccc}
\hline Operation & Expression & Times $(\mathrm{ms})$ \\
\hline$T_{h}$ & $h(\cdot)$ & 0.03 \\
$T_{d}$ & $E_{k}(\cdot) / D_{k}(\cdot)$ & 0.12 \\
$T_{p a}$ & $a_{i} P+b_{i} P$ & 0.18 \\
$T_{p m}$ & $a_{i} P$ & 20.23 \\
$T_{a e}$ & $E_{a e}$ & 34.99 \\
$T_{a d}$ & $E_{a d}$ & 34.78 \\
$T_{\text {exp }}$ & $g^{a_{i}} \bmod n$ & 25.27 \\
$T G_{e}$ & $e(a P, b Q)=e(P, Q)^{a b}$ & 25.64 \\
\hline
\end{tabular}

Table 3 Comparisons of the security features among different protocols

\begin{tabular}{|c|c|c|c|c|c|}
\hline $\begin{array}{l}\text { Related } \\
\text { Protocol }\end{array}$ & Smart meters & Service providers & Trusted Authority & Total costs & $\begin{array}{c}\text { Total } \\
\operatorname{costs}(\mathrm{ms})\end{array}$ \\
\hline Tsai et al.[17] & $4 T_{p m}+T_{e x p}+5 T_{h}$ & $\begin{array}{c}2 T G_{e}+3 T_{p m}+ \\
T_{\text {exp }}+5 T_{h}\end{array}$ & - & $\begin{array}{c}7 T_{p m}+2 T_{e x p}+ \\
2 T G_{e}+10 T_{h}\end{array}$ & 243.73 \\
\hline $\begin{array}{l}\text { Zhang et } \\
\text { al.[18] }\end{array}$ & $T_{p m}+T_{a e}+T_{d}+T_{h}$ & $\begin{array}{c}T_{p m}+T_{a d}+ \\
3 T_{d}+T_{h}\end{array}$ & - & $\begin{array}{c}2 T_{p m}+T_{a e}+ \\
T_{a d}+4 T_{d}+2 T_{h}\end{array}$ & 110.77 \\
\hline $\begin{array}{l}\text { K. Mahmood } \\
\text { et al.[19] }\end{array}$ & $4 T_{p m}+4 T_{h}+T_{p a}$ & - & $T_{p m}+T_{h}$ & $5 T_{p m}+5 T_{h}+T_{p a}$ & 101.48 \\
\hline $\begin{array}{l}\text { Wang et } \\
\text { al.[29] }\end{array}$ & $\begin{array}{c}T G_{e}+3 T_{p m}+ \\
7 T_{h}+T_{d}\end{array}$ & $\begin{array}{c}T G_{e}+3 T_{p m}+ \\
T_{e x p}+7 T_{h}+T_{d}\end{array}$ & - & $\begin{array}{c}2 T G_{e}+6 T_{p m}+ \\
T_{e x p}+14 T_{h}+2 T_{d}\end{array}$ & 198.59 \\
\hline $\begin{array}{l}\text { Ghosh et } \\
\text { al.[30] }\end{array}$ & $\begin{array}{c}T_{p m}+2 T_{e x p}+ \\
T G_{e}+5 T_{h}\end{array}$ & $\begin{array}{c}3 T_{p m}+T_{e x p}+ \\
T G_{e}+4 T_{h}\end{array}$ & - & $\begin{array}{c}4 T_{p m}+3 T_{e x p}+ \\
2 T G_{e}+9 T_{h}\end{array}$ & 208.28 \\
\hline Ours & $2 T_{p m}+12 T_{h}$ & $2 T_{p m}+8 T_{h}$ & $5 T_{h}$ & $4 T_{p m}+25 T_{h}$ & 81.67 \\
\hline
\end{tabular}




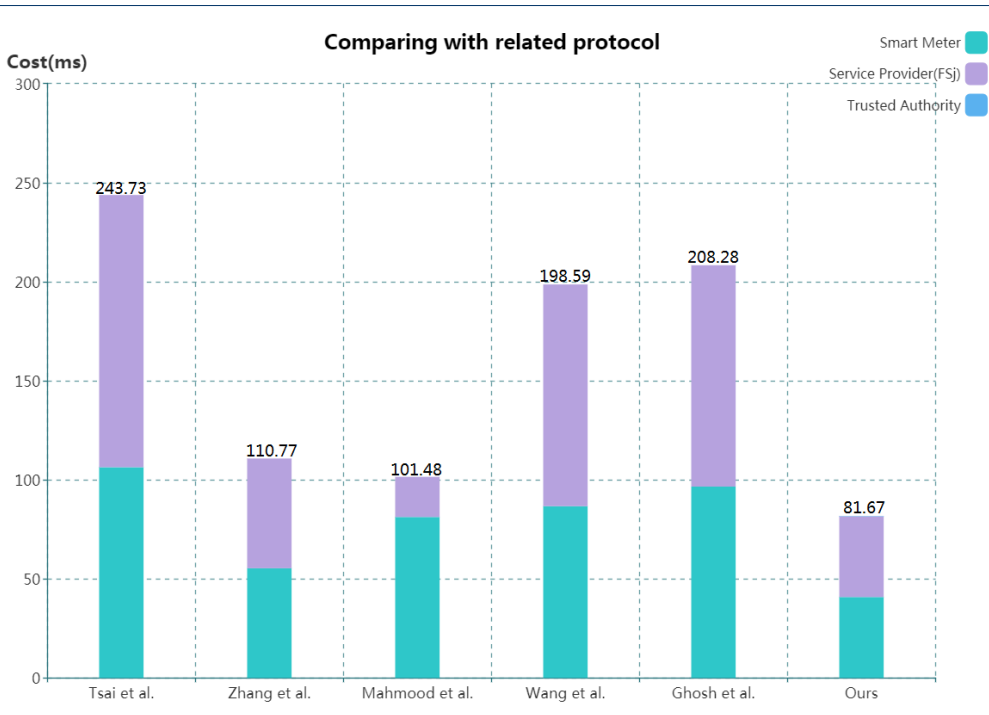

Figure 13 Comparison with related protocol

\section{Conclusion}

In this study, we proposed the use of edge computing to solve the current security issues encountered in SGs. We designed a secure key exchange and mutual authentication protocol based on edge computing for such grids. To verify the security of our proposed protocol, we analyzed the protocol using the automatic tool ProVerif and BAN logic to prove that it can resist various types of attacks. We also compared our proposed protocol with other protocols used in SGs without an edge computing infrastructure. We concluded that the computation time of our proposed protocol is lower than that of other protocols and that the proposed protocol is more secure and lightweight.

List of abbreviations

SG: smart grid; SM: smart meter; SP: service provider; CC: control center; ECC: curve cryptosystem;

Availability of data and materials

Not applicable.

Competing interests

The authors declare that they have no competing interests.

Author's contributions

Conceptualization, Chien-Ming Chen and Lili Chen; Formal analysis, Yanyu Huang, Jimmy Ming-Tai Wu; Methodology, Chien-Ming Chen, Lili Chen and Yanyu Huang; Software, Sachin Kumar; Supervision, Chien-Ming Chen, Jimmy Ming-Tai Wu; Validation, Yanyu Huang; Writing, Jlmmy Ming-Tai Wui.

Acknowledgements

Not applicable.

Funding

Not applicable.

Author details

${ }^{1}$ Shandong University of Science and Technology, Shandong, China. ${ }^{2}$ Harbin Institute of Technology (Shenzhen),

Shenzhen, China. ${ }^{3}$ Ajay Kumar Garg Engineering College, Ghaziabad, India. 
References

1. Zahoor, S., Javaid, N., Khan, A., Ruqia, B., Muhammad, F.J., Zahid, M.: A cloud-fog-based smart grid model for efficient resource utilization. In: 2018 14th International Wireless Communications \& Mobile Computing Conference (IWCMC), pp. 1154-1160 (2018). IEEE

2. Gungor, V.C., Sahin, D., Kocak, T., Ergut, S., Buccella, C., Cecati, C., Hancke, G.P.: Smart grid technologies: Communication technologies and standards. IEEE transactions on Industrial informatics 7(4), 529-539 (2011)

3. Fang, X., Misra, S., Xue, G., Yang, D.: Smart grid:the new and improved power grid: A survey. IEEE communications surveys \& tutorials 14(4), 944-980 (2011)

4. Amin, S.M., Wollenberg, B.F.: Toward a smart grid: power delivery for the 21st century. IEEE power and energy magazine 3(5), 34-41 (2005)

5. Ipakchi, A., Albuyeh, F.: Grid of the future. IEEE power and energy magazine 7(2), 52-62 (2009)

6. Khurana, H., Hadley, M., Lu, N., Frincke, D.A.: Smart-grid security issues. IEEE Security \& Privacy 8(1), 81-85 (2010)

7. Bonomi, F., Milito, R., Zhu, J., Addepalli, S.: Fog computing and its role in the internet of things. In: Proceedings of the First Edition of the MCC Workshop on Mobile Cloud Computing, pp. 13-16 (2012)

8. Stojmenovic, I., Wen, S.: The fog computing paradigm: Scenarios and security issues. In: 2014 Federated Conference on Computer Science and Information Systems, pp. 1-8 (2014). IEEE

9. Hu, P., Dhelim, S., Ning, H., Qiu, T.: Survey on fog computing: architecture, key technologies, applications and open issues. Journal of network and computer applications 98, 27-42 (2017)

10. Hu, P., Ning, H., Qiu, T., Song, H., Wang, Y., Yao, X.: Security and privacy preservation scheme of face identification and resolution framework using fog computing in internet of things. IEEE Internet of Things Journal 4(5), 1143-1155 (2017)

11. Deng, R., Lu, R., Lai, C., Luan, T.H.: Towards power consumption-delay tradeoff by workload allocation in cloud-fog computing. In: 2015 IEEE International Conference on Communications (ICC), pp. 3909-3914 (2015). IEEE

12. Okay, F.Y., Ozdemir, S.: A fog computing based smart grid model. In: 2016 International Symposium on Networks, Computers and Communications (ISNCC), pp. 1-6 (2016). IEEE

13. Hou, X., Li, Y., Chen, M., Wu, D., Jin, D., Chen, S.: Vehicular fog computing: A viewpoint of vehicles as the infrastructures. IEEE Transactions on Vehicular Technology 65(6), 3860-3873 (2016)

14. Truong, N.B., Lee, G.M., Ghamri-Doudane, Y.: Software defined networking-based vehicular adhoc network with fog computing. In: 2015 IFIP/IEEE International Symposium on Integrated Network Management (IM), pp. 1202-1207 (2015). IEEE

15. Fouda, M.M., Fadlullah, Z.M., Kato, N., Lu, R., Shen, X.S.: A lightweight message authentication scheme for smart grid communications. IEEE Transactions on Smart grid 2(4), 675-685 (2011)

16. Saputro, N., Akkaya, K., Uludag, S.: A survey of routing protocols for smart grid communications. Computer Networks 56(11), 2742-2771 (2012)

17. Tsai, J.-L., Lo, N.-W.: Secure anonymous key distribution scheme for smart grid. IEEE transactions on smart grid 7(2), 906-914 (2015)

18. Zhang, L., Tang, S., Luo, H.: Elliptic curve cryptography-based authentication with identity protection for smart grids. PloS one 11(3), 0151253 (2016)

19. Mahmood, K., Chaudhry, S.A., Naqvi, H., Kumari, S., Li, X., Sangaiah, A.K.: An elliptic curve cryptography based lightweight authentication scheme for smart grid communication. Future Generation Computer Systems 81, 557-565 (2018)

20. Hassan, R., Radman, G.: Survey on smart grid. In: Proceedings of the IEEE SoutheastCon 2010 (SoutheastCon), pp. 210-213 (2010). IEEE

21. Ericsson, G.N.: Cyber security and power system communication-essential parts of a smart grid infrastructure. IEEE Transactions on Power Delivery 25(3), 1501-1507 (2010)

22. Kim, S., Kwon, E.Y., Kim, M., Cheon, J.H., Ju, S.-h., Lim, Y.-h., Choi, M.-s.: A secure smart-metering protocol over power-line communication. IEEE Transactions on Power Delivery 26(4), 2370-2379 (2011)

23. Nazmudeen, M.S.H., Wan, A.T., Buhari, S.M.: Improved throughput for power line communication (plc) for smart meters using fog computing based data aggregation approach. In: 2016 IEEE International Smart Cities Conference (ISC2), pp. 1-4 (2016). IEEE

24. Chien, H.-Y., Chen, C.-H.: Mutual authentication protocol for rfid conforming to epc class 1 generation 2 standards. Computer Standards \& Interfaces 29(2), 254-259 (2007)

25. He, D., Kumar, N., Lee, J.-H., Sherratt, R.S.: Enhanced three-factor security protocol for consumer usb mass storage devices. IEEE Transactions on Consumer Electronics 60(1), 30-37 (2014)

26. Moosavi, S.R., Gia, T.N., Nigussie, E., Rahmani, A.M., Virtanen, S., Tenhunen, H., Isoaho, J.: End-to-end security scheme for mobility enabled healthcare internet of things. Future Generation Computer Systems 64, 108-124 (2016)

27. Wazid, M., Das, A.K., Kumar, N., Vasilakos, A.V.: Design of secure key management and user authentication scheme for fog computing services. Future Generation Computer Systems 91, 475-492 (2019)

28. Bruno, B., Smyth, B., Cheval, V.: ProVerif 1.93: Automatic cryptographic protocol verifier, user manual and tutorial. Inria (2016)

29. Wang, Y.: Password protected smart card and memory stick authentication against off-line dictionary attacks. In: IFIP International Information Security Conference, pp. 489-500 (2012). Springer

30. Ghosh, D., Li, C., Yang, C.: A lightweight authentication protocol in smart grid. IJ Network Security 20(3), 414-422 (2018) 